Historic, Archive Document

Do not assume content reflects current scientific knowledge, policies, or practices. 
J. W. GODDARD

\section{Established 1914}

\section{Circular and Price List of Southern Médientate Plánts}

I am prepared to supply many varieties of medical and wild flowering plants from this locality. I will be pleased to give my customers any information I can in regard to any plant in this list.

\section{Common Name \\ Latin Name \\ Price per Doz.}

1. Wild Yam-Dioscorea Villosa

$\$ .50$

2. Carrion Flower-Smilax Herbacea

1.00

3. Wake Robin-Trillium Sessile.

.50

4. Wake Robin-Trillium Grandiflorum

.50

5. Bath Flower-Trillium Erectum

.50

6. Indian Cucumber-Medeola Virginica

7. Dog Tooth Violet-Erythronium Americanum

8. Squill-Scilla Esculenta

1.00

.25

1.50

9. Star of Bethlehem-Ornithogalum Umbellatum

10. False Solomon Seal-Smilacina Racemosa

11. Bellwort-Uvularia Grandifloria

12. False Hellebore-Veratrum Viride.

13. Spiderwort-Tradescantia Virginica

14. Nightshade-Solanum Nigrum

15. Bittersweet-Solanum Durcamara

16. Horsenettle-Solanum Carolinensis

17. Apple of Peru-Nicandra Physaloides

18. Groundcherry-Physalis Viscosa

19. Thornapple-Datura Stramonium

20. Columbo Root-Frasera Carolinensis

21. Dog Bane-Apocynum Androsaemifollum

22. Nilkweed-Asclepias Cornutil.

23. Milkweed-Asclepias Incarnata

24. Milkweed-Asclepias Quadrifolla

25. Butterfly Weed-Asclepias Tuberosa.

26. Virginia Snakeroot-Aristolochia Serpentaria

27. Marvel of Peru-Mirabilis Jalapa..

28. Yellow Sorrel-Rumex Crispus.

29. Sheep Sorrel-Rumex Acetosella

30. Goose Foot-Chenopodium

31. Common Goose Foot-Chenopodium Album

32. Oak of Jerusalem-Chenopodium

33. Wood Nettle-Lapotretea Canadensis.

34. Richweed-Pilea Pumilla

35. Green Dragon-Arisaema Dracontium.

36. Cattail-Typha Latifolia

.50

.50

.50

1.00

.50

.50

.50

.50

(Seed $\$ 1.50$ per 1000 )

(Seed 1.50 per 1000 )

(Seed .50 per 1000 )

.50

.50

.50

.50

.50

1.00

.50

(Seed $\$ 1.50$ per 1000 )

.50

.50

(Seed

(Seed

(Seed

.50

(Seed

2.00

.50

$\begin{array}{ll}1.50 & 12.00 \\ 1.50 & 12.00 \\ 1.50 & 12.00 \\ 1.50 & 12.00 \\ 1.50 & 12.00 \\ 5.00 & 30.00 \\ 1.50 & 12.00\end{array}$

$\begin{array}{ll}1.00 & 10.00\end{array}$

$\begin{array}{ll}1.00 & 10.00\end{array}$

.50 per 1000 )

.50 per 1000$)$

.50 per 1000$)$

1.50

12.00

.50 per 1000 )

8.00

1.50 
Common Name

Latin Name

Price per Doz.

By Mail

Express

Arrowhead-Sagittaria Variabilis

1.00

1000

37. Arrowhead-Sagittaria

1.00

3.00

1000

39. No English-Clematis Virginiana

5.00

3.00

40. Leather Flower-Clematis Viorna

5.00

35.00

41. Wind Flower-Anemone Nemorosa

.50

35.00

42. Liverwort-Hepatica Acutiloba

43. Liverwort-Hepatica Triloba

1.50

12.00

2.00

44. Meadow Rue-Thalictrum Cornuti.

(Seed $\$ 1.00$ per 1000 )

45. Columbine-Aquilegia Canadensis

46. Larksspur-Delphinium Tricorne

.75

2.00

18.00

(Seed $\$ 2.00$ per 1000 )

47. Moonseed-Menispermum Canadense

48. Pappose Root-Caulophyllum Thalietroides.

49. Twinleaf-Jeffersonia Diphylla

50. Squirrel Corn-Dicentra Canadensis.

51. Winter Cress-Barbarrea Vulgaris

52. Black Mustard-Brassica Nigra.

53. Horseradish-Amorcia American

54. Bluecurls-Brunella Vulgaris

55. Skullcap-Scutellaria Galericul

55. Hoarhound-Marrubium Vulgare

57. Houndtongue-Cynoglossum Morrisoni

58. Comfrey--Symphytum Offeinale

59. No English-Phlox Maculata

60. Greek Valerian-Polemonium Reptans.

61. Green Violet-Solea Concolor

62. Violet-Cucullata

63. Violet-Pubescens

64. Violet-Canadensis

65. Violet-Striata

66. St. John's Wort-Hypericum Prolificum.

67. Mouse Ear-Cerastium Bulgatum

68. Spring Beauty-Claytonia Virginica

69. Hollyhock-Althaea Rosea

70. Mallow-Malva Rotundifolia

71. Touch-me-Not-Impatiens Pallida

72. Touch-me-Not-Impatiens Fulva

73. Staff Tree-Celastrus Scandens

74. Milkwort-Polygala

75. Senna-Cassia Marilandica

75. Bush Tree Foil-Desomodium

77. Bush Clover-Lespedeza Capitata.

78. Cinque Foil-Potentilla Canadensis.

79. Agrimony-Agrimonia Eupatoria

80. Stone Crop-Sedum Fernatum

81. Nightshade-Circaea Lutetiana

82. Sweet Cicely--Osmorhiza Longistylis

83. Sweet Cicely-Osmorhiza Brevistylis

84. Golden Alexander-Carum Aureum
.50

1.00

.50

.50

.50

(Seed

\section{.50}

.50

.50

$\begin{array}{ll}1.00 & 12.00\end{array}$

$3.00 \quad 25.00$

$1.50 \quad 12.00$

$1.50 \quad 12.00$

$1.50 \quad 12.00$

.50 per 1000)

1.50

1.50

1.50

12.00

1.50

12.00

(Seed .50 per 1000 )

$\begin{array}{ll}.50 & 1.50\end{array}$

$\begin{array}{ll}.50 & 1.50\end{array}$

1.00

.75

.50

.50

.50

.50

.50

.50

.25

1.00

.50

(Seed

(Seed

\section{.50}

.50

(Seed

.50

1.00

.50

.50

$.5 n$

.50

2.00

.50

.50
1.50
3.00

2.00

1.50

1.50

1.50

1.50

1.25

1.50

1.25

3.00

1.25

.50 per $100 n$ )

.50 per 1000 )

$\begin{array}{ll}2.00 & 15.00 \\ 1.25 & 10.00\end{array}$

10.00

2.00 per 1000 )

1.50

3.00

1.50

1.50

1.50

1.50

10 กó

1.50

1.50
12.00

10.00

12.00

1n $n$ ?

10.00

12.00 12.00 


\section{J. W. GODDARD}

Stone Mountain, Ga.

T'wo year old Ginseng Plants

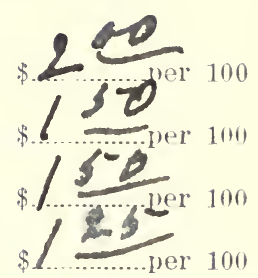

One year old cinseng f'lants

T'wo year old Golden Seal Plants

One year old Golden Seal Plants

Ginseng seed
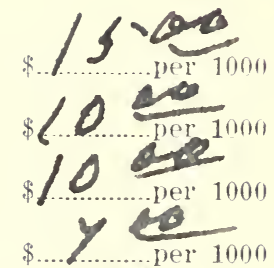
$15^{-0}$

1 per 1009

Golden seal seed

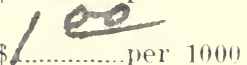

Pink Root seed

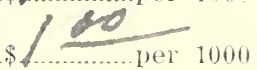

Write for Prises on other Sccels and Plants 
1. $r$ 
86. Water Hemlock-Cicuta

87. Spikenard-Aralia Racemosa

1.00

1.50

Express

89. Spikenard-Aralia Spinosa

1.00

3.00

1000

90. Fever Wort-Triosteum

3.00

91. Cleavers-Galium (Several Varieties)

1.00

92. Partridge Berry-Mitchella Repens

.50

.50

.50

93. Boneset-Eupatorium (Several Varieties)

.50

95. Fleabane-(Several Varieties)

96. Sunflower-(Several Varieties)

97. Chamomile-Anthemis

98. Tansy-Tanacetum Vulgare

99. Everlastino-Antennaria Margarita

100. Lionfoot-Nabalus Albus

1.01. Cardinal Flower-Lobelia Cardinalis.

(Seed

3.00

102. Lobelia-Lobelia Syphilitica

103. Indian Tobacco-Lobelia Inflata

104. Beechdrops-Epiphegus Virginiana

105. Mullen-Verbascum

106. Speedwell-Veronica

107. Culver's Root-Veronica Virginica

108. Wool-mouth-Dasystoma Flava

1.09. Vervain-Verbena (Several Varieties)

110. Bee Balm-Melissa

.50

.50

.50

.50

(Seed $\$ 1.00$ per 1000 )

1.50

3.00

1.25

1.25

1.25

12.00

1.50

10.00

10.00

111. Mountain Mint-Monarda

.50

(Seed

.50

.50 per 1000 )

10.00

$\begin{array}{ll}1.50 & 10.00\end{array}$

$1.50 \quad 10.00$

$1.50 \quad 10.00$

$1.50 \quad 10.00$

112. Blue Flag-Iris

5.00

113. Potty Root-Aplectrum Spicatum

114. Rattle Snake Planting-Epipactus Tesselald

115. Catnip-Nepata Cataria

116. Star Grass-Aletris farinosa

117. White cohosh-Actaea alba

118. Acorus (calamus)-Sweet flag..............

11.9. Wild Turnip-Arisaema triphyllum.

120. Burdock-Lappa officiualis

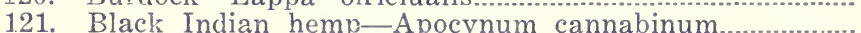

122

Black Indian hemp-Apocynum cannabinum

Monkshood-Aconitum napellus

1.50

.50 per 1000 )

( Seed $\quad .50$ per 1000 )

.50

(Seed

1.50

.50

.50 per 100 )

.50

3.00

1.50

2.00

.50

.50

.50

1.25

$3.0 n$

1.00

4.50

.60

60

1.60

1.60

1.50

.40

.50

.50

.50

.60

$.5 n$

123.

American angelic-Archangelica atropurpurea

.60

.60

Star root-Luteum-Shamaclirium

.50

.50

125.

Black snakeroot-Cimicifuga racemosa

.50

127.

Stoneroot-Colliusonia-Canadensis

1.60

1.60

1.50

1.75

150

1.60

1.60

1.50

1.50

1.50

1.00

128. Jimson weed-Datura stranomum

60

129 .

Button Snakeroot-Laciniaria scariesa

130. Yellow jasamine-Gelsemium sempervireu

131. Cranesbill-Geranium maculatum

132. Day lily-Hemerocallis fulva.

133. Sneeze weed-Helenium autumnale.

.50

5.00

1.50

12.00

1.50

10.00

1.50

35.00

12.00

12.00

10.00

1.50

10.00

10.00

10.00

11.00

10.00

10.00

11.00

10.00

10.00

10.00

1.50

10.00

1.50

10.00

10.00

10.00

10.00

10.00 
Common Name

Latin Name

Price per Doz.

By Mail

134. Turks cap lily-Lilium superbum

100

135. Solomon seal-Polygonatum billorum

.90

5.00

Express

1000

136. Senega snakeroot-Pollygala senega

.50

1.50

10.00

137. Mandrake-Podophyllum - peltatum .............................

1.65

11.00

1.50

10.00

138. Phytolacca-Decandra poke

1.50

10.00

139. Moss pink-Phlox subulata.

1.50

10.00

140. Bloodroot-Canadensis sanguinaria.............................

1.50

10.00

141. Pinkroot-Spigelia-Marsiandica

1.60

12.00

142. Fier pink-Silene virginica

1.60

12.00

143. Queen's delight_-Stillingia sylvatica

1.60

15.00

144. Soap root-Saponaria officinalis

1.50

11.00

145. False Solomon seal-Smilacina stellata............................... $\quad .50$

1.50

10.00

146. Blue myrtle-Vinca minor.

1.50

1.40

10.00

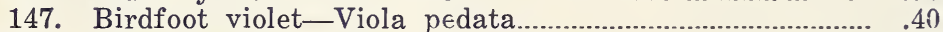

148. Downy yellow violets-Viola pubescens.............................. $\quad .50$

149. Common violets_-Viola cucullata_........................................ $\quad .40$

1.40

9.00

1.30

9.00

1.30

8.00

150. Yellow dock-Rumex crispus

.40

151. Bear grass-Yucca Aloefolia.

.50

1.50

8.00

152. Bottle gentians-Gentiana andrewaii

.95

2.50

1.80

153. Queen-of-the-meadow-Eupatorium purpureum ........... $\quad .75$

2.00

154. Maiden hair fern-Adiantum pedatum....

2.00

5.00

3.00

156. Tiger lily-Lilium tigrinum

.75

1.50

158. Wild Strawberry-Fragaria virginia

1.00

.50

1.75

159. Wild Ginger-Asarum canadense.................... Spotted Wintergreen-Chimaphila maculata.

.75

1.50

I can supply seeds from many of the above plants in this list, also tree seeds, Ginseng and Golden Seal seeds and roots. A certificate of inspection will accompanv all shipments of plants. If for any cause I cannot fill your order during the season, I will return your money.

Make all orders payable to

J. W. GODDARD,

R. D. No. 3, Stone Mountain, Ga. 